\title{
El Darriwiliense inferior (Ordovícico Medio) en el extremo norte de la Precordillera de Mendoza, Argentina: implicaciones estratigráficas y bioestratigráficas
}

\author{
M.S. Beresi(1), O.L. Bordonaro(1), S.E. Heredia ${ }^{(2)}$, A. Mestre ${ }^{(2)}$ y B.A. Toro ${ }^{(3)}$ \\ (1) CONICET-IANIGLA, CCT Mendoza, Av. Ruiz Leal s/n, 5500. Mendoza, Argentina. \\ mberesi@mendoza-conicet.gob.ar
}

(2) CONICET-CIGEOBIO-Instituto de Investigaciones Mineras. Universidad Nacional de San Juan. Av. Libertador y Urquiza, 5400 San Juan Argentina.

sheredia@unsj.edu.ar; amestre@unsj.edu.ar

(3) CICTERRA-CONICET, Universidad Nacional de Córdoba, Av. Vélez Sarsfield 1611, X5016 GSA, Córdoba, Argentina.

btorogr@mendoza-conicet.gov.ar

\begin{abstract}
RESUMEN
En esta contribución se da a conocer una nueva localidad fosilífera en la Cantera Los Guanacos del extremo norte de la sierra de Las Higueras, a $10.5 \mathrm{~km}$ de la localidad de Salagasta, Precordillera de Mendoza, oeste de Argentina. Se trata de un nivel carbonático lenticular fosilífero intercalado entre la unidad mixta pelítica/ carbonática (transfacies) correlacionable con la base de la Formación Los Azules. Éste se caracteriza por un límite erosional de espesor milimétrico y por una distribución mecánica de bioclastos e intraclastos, por encima del mudstone basal oscuro. La capa bioclástica se interpreta como el depósito de un evento tempestítico acaecido en la plataforma carbonática. Se determinan las microfacies carbonáticas y las asociaciones de macro y microfauna. Se registran y describen los trilobites Mendolaspis salagastensis y especies indeterminadas de Agnostidae, Illaenidae, Leiostegiidae, Encrinuridae, Telephinidae y Trinucleidae. Se reconocieron braquiópodos indeterminados de la familia Obolidae. Los graptolitos son comparables con Levisograptus cf. austrodentatus y en base a la fauna de conodontos se reconoce la Zona de Yangtzeplacognathus crassus de edad darriwiliense temprana (Ordovícico Medio). Se discuten las implicaciones bioestratigráficas de estos registros, que permiten la correlación de estos niveles de la Precordillera de Mendoza con otras regiones de la Precordillera de San Juan, del Noroeste argentino y del mundo. Se brindan además algunas consideraciones paleoambientales surgidas del análisis de estos registros faunísticos y de las microfacies carbonáticas.
\end{abstract}

Palabras clave: Argentina, Darriwiliense, conodontos, graptolitos, trilobites

\section{The lower Darriwilian (Middle Ordovician) at the northern extreme of the Mendoza Precordillera, Argentina: stratigraphic and biostratigraphic implications}

\author{
ABSTRACT
}

A tectonically affected Middle Ordovician succession crops out at the Los Guanacos quarry in the sierra de Las Higueras, in the Precordillera of Mendoza, western Argentina. This is represented by the San Juan Formation which is transitionally covered by a mixed carbonate-shaly unit of dark colours, 1.5 metres thick (transfacies) which can be interpreted as the base of the Los Azules Formation. A 5-cm thick intra-bioclastic pack/grainstone bed interbedded in the lower part of this unit has been interpreted as a tempestite. It is characterized both by a millimetre-thick erosional boundary, just above the dark mudstone layer and strong mechanical distribution of bioclasts and intraclasts, suggesting sedimentary structures associated with a storm event of high energy possibly occurring in the middle part of the platform. Towards the bottom, a hummocky 
structure thin interval, under the dark mudstone, is interpreted as the record of another tempestite event. The mudstone represents the post-storm event reflecting the low energy of the marine water environment. The lenticular layer contains predominantly lingulid shells, trilobites and graptolites, all highly fragmented, which suggests they have been reworked and subsequently mixed and quickly redeposited during a storm event that caused the mortality of these faunas. The trilobites Mendolaspis salagastensis and indeterminate species of Matagnostidae, Illaenidae, Leiostegiidae, Encrinuridae, Telephinidae and Trinucleidae have been recorded for the first time at this locality. The conodont fauna allows the identification of the Yangtzeplacognathus crassus Zone, which indicates the lower Darriwilian (Middle Ordovician). Graptolites assignable to Levisograptus cf. Levisograptus austrodentatus and Levisograptus sp. are described for the first time in the studied area and biostratigraphic implications validate the early Darriwilian age for the bearing levels. Finally, based on the studied records, correlation with different regions at the Precordillera, northwestern Argentina and worldwide, as well as some paleoenvironmental considerations, are additionally discussed.

Keywords: Argentina, Darriwilian, conodonts, graptolites, trilobites

\section{ABRIDGED ENGLISH VERSION}

\section{Introduction and methods}

The Lower Paleozoic succession studied in this contribution is located about $40 \mathrm{~km}$ to the north of the city of Mendoza, cropping out at the Sierra de Las Higueras, in the northern Precordillera of Mendoza. It is tectonically affected and represented by a narrow strip of limestone, corresponding to the San Juan Formation (Lower-Middle Ordovician), with meridian direction and dip angle at high west. Overlying the San Juan Formation, a transitional unit (1.5m thick) composed of black shale interbedded with thin dark grey limestone (transfacies) is referring to the lower part of the Los Azules Formation at the Los Guanacos quarry, at the northern end of the sierra de Las Higueras.

A five-centimetre-thick lenticular carbonate level, interbedded in the clastic /carbonate unit, overlays the dark mudstone through a millimetre-thick erosional boundary. Bioclastics and intraclasts of different size and composition commonly occur on the basal surfaces of the fossiliferous bed, displaying random orientation. The lenticular layer contains predominantly lingulid shells, fragmented trilobites and incomplete graptolite tubaria, which suggests that they have been reworked, subsequently mixed and quickly redeposited during a storm event that caused the mortality of these faunas.

The main goal of this contribution is to document the tempestite layer and the biostratigraphic implications of the associated macro and microfauna.

The conodont fauna documents the Yangtzeplacognathus crassus Zone, indicating the lower Darriwilian (Middle Ordovician) from the Precordillera Argentina, according to the scheme proposed by Heredia and Mestre $(2011,2013)$. A conodont sample was collected from the bioclastic bed. The sample was dissolved in diluted formic acid, following the procedures described by Stone (1987). The insoluble fraction of the sample was picked over for conodont elements. All the illustrations are SEM digital photomicrographs.

The hand sample was taken to complete the petrographic descriptions. The microfacies analysis was based on the classification of Dunham (1962), as modified by Embry and Klovan (1971), according to depositional textures. Calcareous carbonate microfacies were based on field observations of textures, sedimentary structures and petrographic analysis of polished slabs and thin sections, in order to identify types of carbonates and skeletal remains. Thin sections were examined under a light microscope.

\section{Results and discussion}

Based on the accumulation of shallow marine water fauna in the basal part of the lenticular bed, stratification, different-sized intraclasts, closed-packeted composition and the erosional irregular contact with the underlying dark mudstone, the bioclastic-intraclastic packstone-grainstone bed has been ascribed to a storm event. The basal mudstone was deposited during the post-storm, and reveals the finalization of the storm event and/or back to normal depositional conditions. The hummocky cross stratification interval underlying the dark mudstone has been ascribed to the upper part of another storm sequence, which occurred immediately before. The complete sequence of the tempestite is not preserved in the study section. 
Beresi, M.S., et al., 2017. El Darriwiliense inferior (Ordovícico Medio) en el extremo... Boletín Geológico y Minero, 128 (1): $93-110$

The stratigraphic analysis allows the extension of the Darriwilian basin to the northern sector of the Precordillera of Mendoza, as well as recognizing a major sedimentary change that helps the paleo-reconstruction of the Precordillera Basin.

Lingulid brachiopod shells of varied sizes are the dominant bioclasts, besides fragments of trilobites and graptolites. This fauna inhabited shallower environments, probably in the middle part of the platform affected by the storm action that erosioned, reworked and redeposited it as a bioclastic layer overlaying the mudstone bed.

The trilobite Mendolaspis salagastensis Rusconi (1951) has been recorded for the first time at this new locality of the Precordillera of Mendoza.

The conodont fauna allows the recording of the Yangtzeplacognathus crassus Zone to Darriwilian levels located in the Precordillera of Mendoza, giving a clear biostratigraphical correlation and age control between different areas of this region.

Graptolites assignable to Levisograptus cf. Levisograptus austrodentatus (Harris and Keble, 1932) and Levisograptus sp. are recorded and described for the first time at the Sierra de Las Higueras, Precordillera of Mendoza. Biostratigraphical implications of these records validate an early Darriwilian age for the bearing levels, as well as regional and international correlation. In addition, paleoenvironmental conclusions are also supported by the analyzed material.

\section{Introducción}

En la sierra de Las Higueras, norte de la Precordillera de Mendoza, localizada a unos $40 \mathrm{~km}$ al norte de la ciudad de Mendoza, aflora una sucesión del Paleozoico Inferior representada por una franja angosta de calizas con rumbo meridiano y buzamiento de alto ángulo al oeste, en parte rebatida y plegada, correspondiente a la Formación San Juan (Ordovícico Inferior-Medio), a la que suprayace una unidad mixta compuesta por calizas delgadas de color gris oscuro intercaladas con pelitas negras (Fig. 1).

El único antecedente previo de estudio de esta sierra era su extremo sur, donde Beresi et al. (1998), Beresi y Rodríguez (2011) y Heredia et al. (2009) describieron dos perfiles estratigráficos en las cercanías de las Canteras 1 y 2 (Fig. 1) y determinaron una asociación de conodontos del Floiense para los tramos superiores de la Formación San Juan. Dichos niveles están asociados a mudstone y wackestone peletoidal y a estructuras sedimentarias de baja energía.

En esta contribución se da a conocer una nueva localidad fosilífera en el extremo norte de la sierra de Las Higueras, a $10,5 \mathrm{~km}$ de la localidad de Salagasta, en la Cantera Los Guanacos. Se analiza un nivel carbonático lenticular intercalado entre la unidad mixta pelítica/carbonática (transfacies) correlacionable con la base de la Formación Los Azules (Fig. 1), donde se estudian la microfacies carbonática y las asociaciones de macro y microfauna. La edad de este nivel basada en conodontos, graptolitos y trilobites es darriwiliense temprano (Ordovícico Medio), constituyendo el primer estudio sistemático de estas faunas en el mencionado lapso temporal para la Precordillera de Mendoza.

\section{Marco geológico}

En la sucesión calcárea-clástica de la región de Salagasta quedan registrados tres ciclos sedimentarios del Paleozoico Inferior que abarcan el lapso comprendido entre el Ordovícico Inferior al Devónico Medio: un ciclo carbonático del Ordovícico y dos ciclos de sedimentación clástica, uno de posible edad silúrica y otro de edad devónica.

Dentro de la región afloran rocas sedimentarias que forman parte de la Provincia Geológica de la Precordillera. En esta serranía se reconocen de oeste a este: sedimentitas recientes y del Grupo Calchaquí del Neógeno, las formaciones Potrerillos, Las Cabras, Río Blanco y Río Mendoza (Grupo Uspallata) del Triásico, la Formación Villavicencio del Devónico Inferior-Medio, la Formación Tambolar? del Silúrico s.l., las formaciones Los Azules y San Juan del Ordovícico Inferior-Medio.

La estructura de la sierra de Las Higueras corresponde en líneas generales a un sinclinal asimétrico amplio, de eje submeridional alabeado, fallado en su flanco oriental. Presenta pliegues y fallas menores orientados paralelamente al eje principal de la estructura y concentrados en los faldeos occidentales de las sierras de las Peñas y de Las Higueras. Los buzamientos del flanco oriental son mayores que los que presenta el flanco occidental del anticlinal.

En la sierra de Las Higueras, hasta la latitud de Salagasta y sobre el flanco oriental del anticlinal, el fallamiento provoca el alzamiento y exposición del contacto Triásico-Devónico y de una franja calcárea de la Formación San Juan con un espesor estimado de $70 \mathrm{~m}$. La disposición estructural de la faja calcárea, limitada en ciertas secciones por dos fallas, genera una respuesta sumamente plástica dando como resultado fenómenos de diapirismo. 


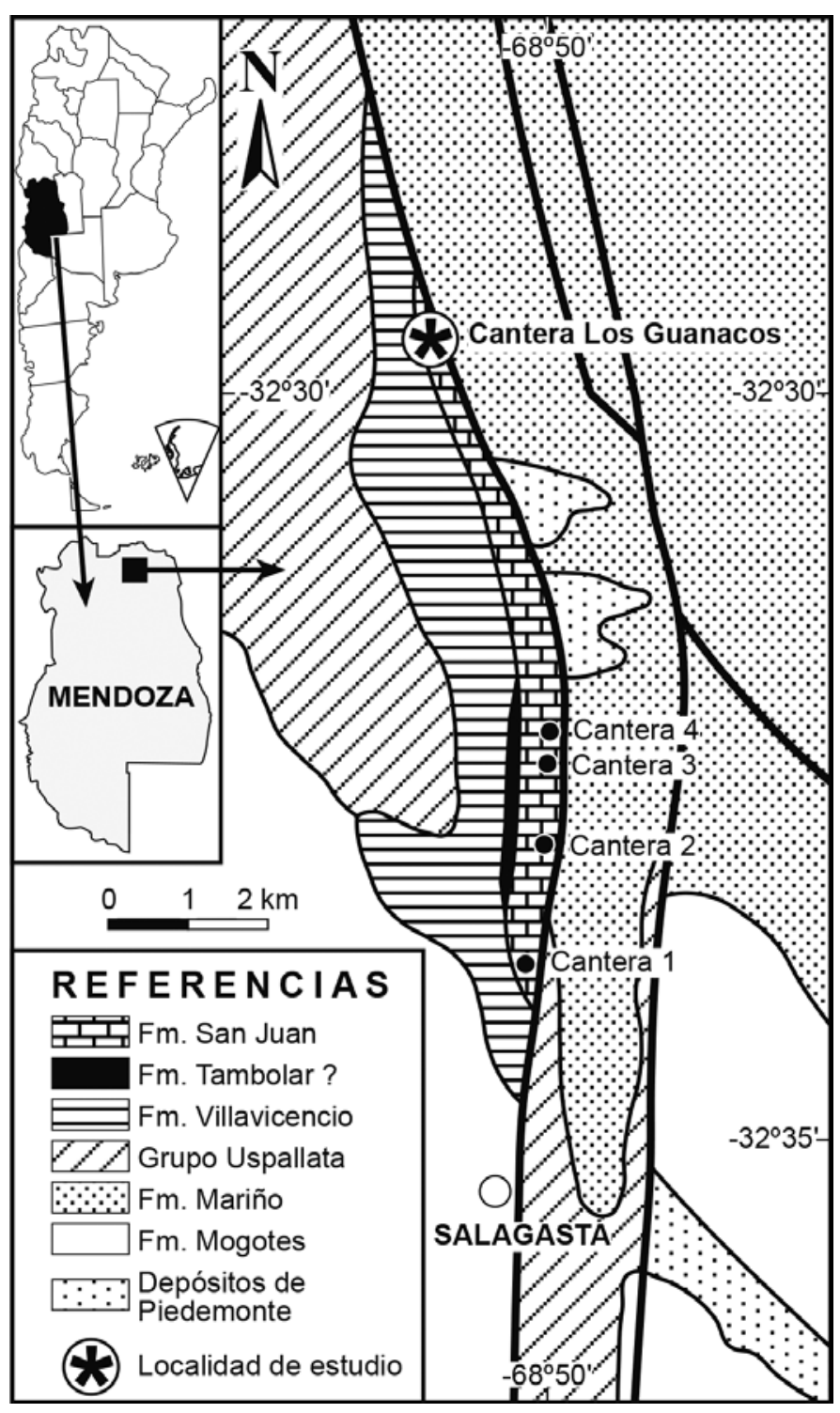

Figura 1. Mapa de ubicación de la sierra de Las Higueras, Precordillera de Mendoza, indicando la sección de estudio.

Figure 1. Location map of the sierra de Las Higueras, Mendoza Precordillera, showing the studied section.

\section{Sección Cantera de Los Guanacos}

En la sección de la Cantera Los Guanacos (Beresi et al., 2014), las calizas de la Formación San Juan tienen $70 \mathrm{~m}$ de espesor máximo (Fig. 2). Mediante un contacto transicional pasan a una unidad mixta calcáreo-pelítica de color gris oscuro a negro finamente estratificada que alcanza los 1,50 m de espesor máximo equivalente a la base de la Formación Los Azules ("Transfacies" de Baldis and Beresi, 1981). Sobre esta unidad, afloran $37 \mathrm{~m}$ de espesor de limolitas moradas y verdes con concreciones, pero sin contenido de macrofósiles que podría corresponder al Silúrico sensu lato, como fuera postulado por Heredia et al. (2010). A continuación, se apoyan bancos de areniscas castañas y por encima grauvacas y pelitas verde oscuras de la Formación Villavicencio de edad devónica inferior-media.

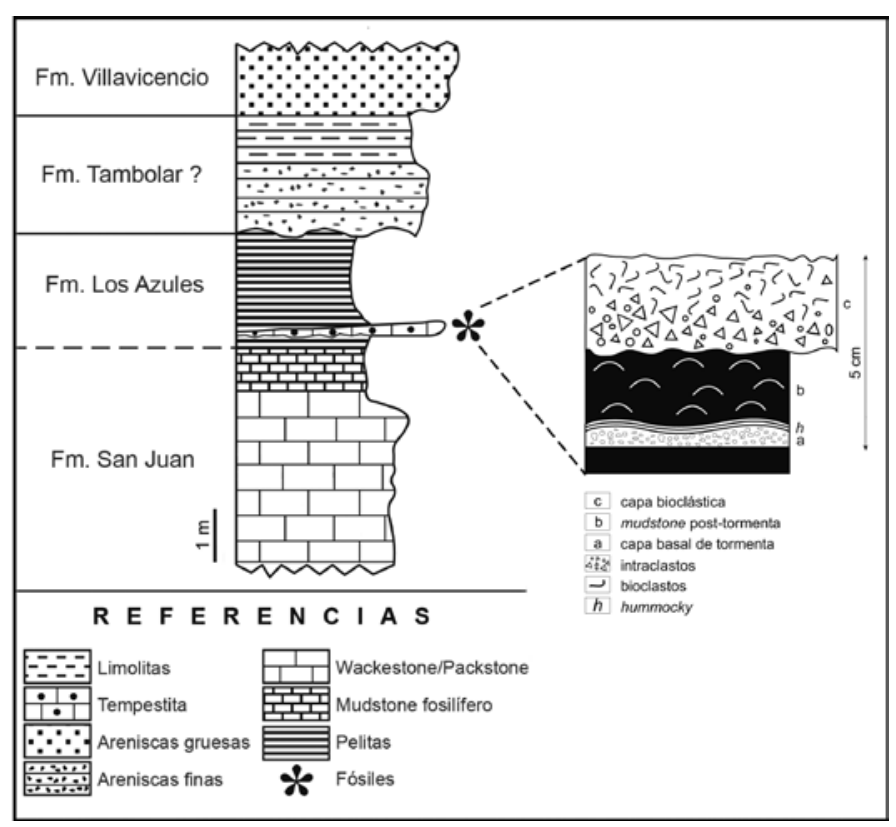

Figura 2. Perfil de la cantera Los Guanacos, extremo norte de Salagasta, sierra de Las Higueras, Precordillera de Mendoza. Se representa de forma esquemática el techo de la Formación San Juan y las formaciones suprayacentes. En detalle, la capa tempestítica y litologías asociadas.

Figure 2. The Los Guanacos quarry section, northern part of the Salagasta, sierra de Las Higueras, Mendoza Precordillera. Schematic drawing of the top of the San Juan Formation and overlying formations. In detail, the tempestite and associated lithologies.

\section{Descripción de la capa bioclástica lenticular}

En la Cantera Los Guanacos la unidad mixta calcáreo-pelítica que sobreyace a las calizas de la Formación San Juan tiene un espesor aproximado de $1,50 \mathrm{~m}$. En esta unidad se intercala un nivel carbonático de forma lenticular de $5-6 \mathrm{~cm}$ de espesor, color gris a castaño oscuro con tonalidades amariIlentas por oxidación, compuesto por un nivel basal de mudstone (1,2 cm de espesor) pardo oscuro, de textura homogénea, con escasas valvas finas desarticuladas de braquiópodos liguliformes y una capa bioclástica-intraclástica de textura pack/grainstone (3,5-4 cm de espesor) de color castaño oscuro con parches de micrita gris oscura y relleno de esparita blanquecina. El pack/grainstone apoya mediante una 

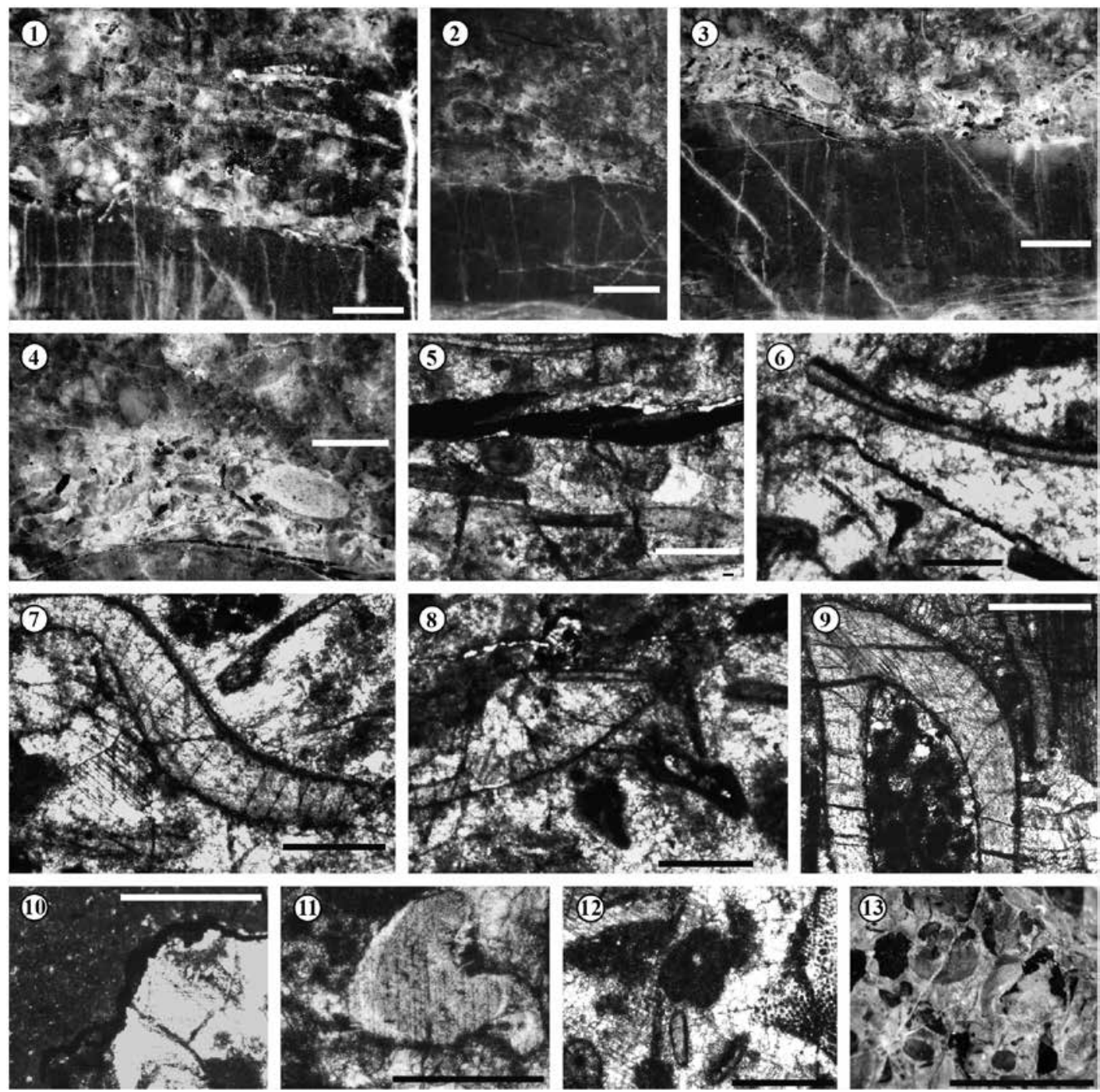

Figura 3. 1-13, Tempestita de la cantera Los Guanacos, Salagasta.1, contacto erosivo entre mudstone y el packstone-grainstone; 2 y $\mathbf{3}$, detalle del contacto erosivo entre las dos unidades. A la base del mudstone intervalo con estratificación tipo hummocky. El mudstone muestra fina bioturbación vertical; 4, intraclastos de diversos tamaños y pequeños fragmentos en cemento de esparita, a la base de la capa bioclástica. En el contacto una valva de braquiópodo linguliforme con la concavidad hacia abajo; 5-12, Secciones delgadas del packstone-grainstone bioclástico; 5, se observan fragmentos de valvas desarticuladas de braquiópodos fosfáticos y una placa de crinoideo; 6, sección longitudinal de una valva de braquiópodo linguliforme; 7,9 y 12, restos de trilobites; 8 , valva articulada de braquiópodo y fragmentos fósiles con recubrimiento de micrita en cemento esparítico con parches de micrita peletoidal; 13 , a la izquierda parte de un trilobite, a la derecha fragmento de crinoideo? y en el centro sección transversal de Nuia y pequeñas valvas de braquiópodos; 9, fragmento de trilobite con parches de matriz peletoidal; 10, contacto erosivo milimétrico con relleno de óxidos de hierro entre el mudstone y la esparita clara; 11, placa de crinoideo; 12, a la izquierda parte de un trilobite, a la derecha fragmento de crinoideo? y en el centro sección transversal de Nuia y pequeñas valvas de braquiópodos; 13, vista del plano superior de la capa con diversos fragmentos de graptolitos y valvas de braquiópodos linguliformes. Escalas gráficas $=5 \mathrm{~mm}(1,2,3,13)$ y $0.5 \mathrm{~mm}(4,5,6,7,8,9,10,11,12)$

Figure 3. 1-13. Tempestite from the Los Guanacos quarry, Salagasta. 1, show a millimetre erosive contact between the dark mudstone and the bioclastic packstone-grainstone, with fossil fragments in clear sparry cement and dark micrite; 2, 3, shows a detail of erosive contact between both units. At the base of the mudstone, a narrow interval with hummocky stratification. The mudstone shows fine vertical bioturbation; 4, show intraclasts of different sizes and dark fragments at the base of the bioclastic bed in clear sparite cement. In the contact, a linguliform brachiopod valve with the concave side down; 5-12, Bioclastic packstone-grainstone thin sections; 5, show fragments of disarticulated linguliform brachiopods and a crinoid plate, in clear sparry calcite cement; 6 , shows a longitudinal section of a linguliform valve; $\mathbf{7}, \mathbf{9}, \mathbf{1 2}$, trilobite fragments; 8 , view of a rhynconellid brachiopod valve and fossil fragments, surrounded by a coating of dark micrite; 13 , cross-sections of Nuia and small brachiopod valves; 9 , patch of dark peletoidal micrite matrix, between a trilobite; 10, millimetre erosive contact, with filling of oxides of iron between the dark mudstone and clear sparite cement of the bioclastic bed; 11, shows a crinoid plate; 12, trilobite (to the left), crinoid fragment? (to the right), cross-section of Nuia and small brachiopod valves; 13, view of the upper surface of bioclastic layer showing diverse fossil fragments, as dark graptolite and small linguliform valves. Scale bars = $5 \mathrm{~mm}(1,2,3,13)$ and 0.5 $\mathrm{mm}(4,5,6,7,8,9,10,11,12)$. 
Beresi, M.S., et al., 2017. El Darriwiliense inferior (Ordovícico Medio) en el extremo... Boletín Geológico y Minero, 128 (1): $93-110$

superficie erosional abrupta (storm erosion), milimétrica, de trazo estilolítico y color amarillento por oxidación, que se superpone al mudstone oscuro (Fig. 3,1-3).

El notorio cambio entre el mudstone y la capa bioclástica-intraclástica se debe a diferentes condiciones hidrodinámicas y tasas de sedimentación. El tope del mudstone evidencia bioturbación (Fig. 3, 1-3). Por debajo del mudstone se ha preservado un delgado intervalo de 4-5 $\mathrm{mm}$ de espesor, con estratificación ondulada de bajo ángulo tipo hummocky (Fig. 3, 2-3).

El pack/grainstone contiene intraclastos de composición micrítica que presentan colores que varían entre pardo rojizos y castaño oscuro. Exhiben formas ovaladas a irregulares y tamaños entre 0,3 a 3,3 mm. Algunos presentan envoltura micrítica oscura y bordes oxidados mostrando cierta orientación paralela a la superficie de erosión (Fig. 3-4).

En la parte basal del pack/grainstone, se ha preservado una concentración de fósiles (basal skeletal shell concentration) que constituyen una coquina. Los fósiles dominantes (65\%) corresponden a valvas externas completas desarticuladas de braquiópodos linguliformes (Fig. 3, 5-6), cranidios y pigidios de trilobites $(20 \%)$ y escasas valvas de braquiópodos articulados (3\%) (Fig. 7.3). Las valvas muestran una cierta orientación paralela a la superficie de erosión basal, y a menudo la convexidad hacia arriba con relleno micrítico. En proporción muy subordinada se encuentran tubarios de graptolitos $(7 \%)$ pobremente preservados, a veces aplastados y conservando restos de materia orgánica carbonizada, o más comúnmente parcialmente rellenos con pirita, además se encuentran escasas placas de crinoideos $(2 \%)$ y de secciones transversales recristalizadas de Nuia (microorganismo problemático) (Fig. 3.5-12).

\section{Interpretación}

Las tempestitas o capas de tormentas se producen rápidamente a partir de eventos con abundante carga en suspensión y tracción en condiciones de régimen de flujo menguante. Son el producto de eventos aperiódicos de variadas magnitudes (Ager, 1973; cf. Kreisa, 1981). El espesor de estas capas varía entre pocos milímetros a decenas de centímetros a diferencia del espesor de las capas inferiores y superiores. Exhiben amplia variación en el espesor, tamaño del grano y estructuras internas, dependiendo de la proximidad y de la intensidad de las tormentas. De forma similar a turbiditas, el espesor de las capas de tempestitas varía proporcionalmente con el máximo tamaño del grano de la capa. Estas capas son comunes en shallowing-up secuencias (Kreisa, 1981). En sectores proximales de la plataforma es frecuente que las tempestitas se amalgamen.

Los depósitos de tormenta y corrientes están normalmente intercalados en calcipelitas, margas o pelitas y exhiben estratificación ondulada característica tipo HCS.

Para la interpretación de la capa bioclástica de Salagasta, se ha tenido en cuenta la integración de litofacies, biofacies y microfacies (acorde a Dunham, 1962). Las características consideradas son las siguientes: la textura gruesa (pack/grainstone); el contenido de las faunas densamente empaquetadas y $\sin$ selección por tamaños; la presencia de intraclastos retrabajados de variadas litología, colores y tamaños, algunos oxidados y otros con envolturas micríticas; las condiciones paleoecológicas de las faunas depositadas; las variaciones texturales de los carbonatos (de mudstone a pack/grainstone) y el contacto basal erosional de tipo estilolítico, abrupto separando el pack/grainstone que suprayace al mudstone oscuro.

De acuerdo a estas características interpretamos que la capa bioclástica reflejaría un deposito hidrodinámico que puede ser el resultado de la acción de una tormenta, una "tempestita" carbonática (Ager, 1973) producida sobre la plataforma fangosa somera. Este tipo de plataformas con sustrato fangoso, son altamente episódicas por la acción seguida de tormentas de poca duración durante prolongados periodos de buen tiempo (Kreisa, 1981).

Las secuencias tempestíticas completas difícilmente se preserven debido a modificaciones producidas por los efectos de la erosión y bioturbación. En este depósito se ha preservado una capa basal carbonática de textura pack/grainstone con concentración de valvas de lingúlidos lo que puede explicarse por una cercanía del área que constituyó su hábitat natural (cf. Aigner 1982) y por la cantidad de shelly fauna en el área de erosión de la tormenta.

La presencia de intraclastos micríticos y la acumulación de fósiles indican el carácter localmente erosivo del flujo. Considerando estas características y la base erosional, se lo puede interpretar como el registro de una secuencia tempestítica asociado a tormentas originadas en offshore (cf. Aigner, 1982).

Por otra parte, la forma lenticular de la capa carbonática bioclástica indicaría que fue depositada por un flujo unidireccional y que correspondería a un único evento de tormenta posiblemente en sectores medios de una plataforma fangosa. La tormenta interrumpió la depositación del fango carbonático en un ambiente con bajo régimen de energía, caracterizado por lechos barrosos con fauna dominante de braquiópodos linguliformes y trilobites. Por otro lado, la 
estratificación ondulada de tipo hummocky preservada por debajo del mudstone basal posiblemente represente parte de otro evento de tormenta ocurrido con anterioridad al que depositó la capa bioclástica que se describe. El mudstone se interpreta como de post-tormenta (fair weather mud deposition) el cual refleja tanto la finalización de la acción de la tormenta y/o el retorno a la sedimentación normal.

Acorde a lo expuesto, se infiere que la capa bio-intraclastica, el mudstone y el nivel con hummocky, representan el registro incompleto de dos eventos de tormenta que afectaron la plataforma carbonática durante el Darriwiliense inferior-alto en esta parte de la cuenca de Precordillera.

\section{Paleontología}

La fauna está constituida mayoritariamente por valvas desarticuladas de braquiópodos linguliformes $(50 \%)$ y trilobites $(35 \%)$, y en forma muy subordinada tubarios fragmentados y oxidados de graptolitos $(7 \%)$, artejos de crinoideos $(2 \%)$, muy escasas Nuia y fragmentos indeterminables de pequeño tamaño y color negro.

Los trilobites, graptolitos y braquiópodos se hallan depositados en la colección paleontológica de invertebrados del IANIGLA, CCT-Mendoza, CONICET, bajo la sigla IANIGLA-PI. En cuanto a los conodontos, se encuentran registrados en el repositorio del Instituto de Geología Emiliano Aparicio, bajo la sigla INGEO-MP.

\section{Conodontos}

La asociación de conodontos recuperada del nivel carbonático de la base de la Formación Los Azules en la sección estudiada, está conformada por 90 elementos en mal estado de preservación, altamente fragmentados. En la misma se han podido reconocer las siguientes especies: Lenodus variabilis (Sergeeva, 1963), Paroistodus horridus (Barnes and Poplawski, 1973), Periodon macrodentatus (Graves and Ellison, 1941) (Fig.4, 1-4) entre otros. El hallazgo de estas especies permite el registro de la Zona de Yangtzeplacognathus crassus (Beresi et al., 2014) la cual indica una edad darriwiliense temprana (Ordovícico Medio) coincidiendo con el esquema bioestratigráfico propuesto para Precordillera por Heredia and Mestre $(2011,2013)$ y Mestre and Heredia (2013).

En los últimos años, la Zona de $Y$. crassus ha sido intensamente estudiada en varios sectores de la Precordillera de San Juan, ya que en la misma se verifica, especialmente en los sectores orientales de la cuenca, el pase entre la Formación San Juan y la suprayacente Formación Los Azules. Este pase representa un importante cambio en el régimen sedimentario en la cuenca de Precordillera durante el Darriwiliense (Fig. 8) desde rocas carbonáticas de rampa proximal a sedimentitas mixtas clásticas-carbonáticas de rampa media-distal, con gran participación de clásticos finos.

El hallazgo de esta zona en la región más septentrional de la Precordillera mendocina permite extender el límite de la cuenca del Darriwiliense, así como el importante cambio sedimentario acaecido en ese momento hasta este sector de la Precordillera, lo que representaría un cambio significativo en la configuración de la cuenca para el Ordovícico Medio.

Heredia et al. (2009) reconocen la Zona de O. intermedius en el techo de la Formación San Juan en

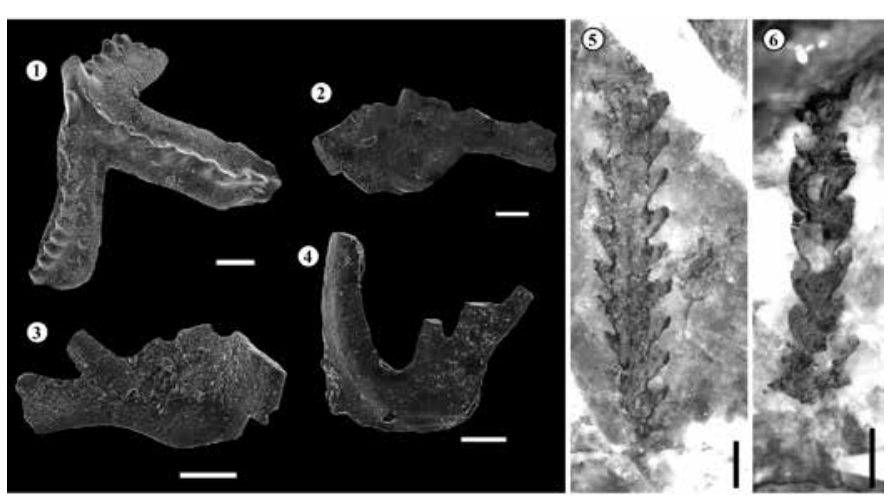

Figura 4. 1-6, Conodontos y graptolitos de la cantera Los Guanacos, Salagasta. 1, Lenodus variabilis (Sergeeva). Elemento $\mathrm{Pb}$, vista anterior, INGEO-MP-5001(1). 2-4, Paroistudus horridus (Barnes and Poplawski). 2, Elemento P, vista lateral, INGEO-MP-5002(1). 3, Elemento $P$, vista lateral, INGEO-MP-5002 (2). 4, Elemento $\mathrm{Sb}$, vista lateral, INGEO-MP-5002 (3). Escala gráfica $=100 \mu \mathrm{m} . \mathbf{5}$, Levisograptus sp. cf. Levisograptus austrodentatus (Harris and Keble); IANIGLA-PI 2979, ejemplar adulto e incompleto, que muestra el septo medio levemente ondulado y las tecas levemente sigmoidales, con doble curvatura. Se encuentra asociado con otro ejemplar correspondiente a un extremo proximal regularmente conservado. 6, Levisograptus sp.; IANIGLA-PI 2980, tubario juvenil e incompleto. Escala gráfica $=1 \mathrm{~mm}$.

Figure 4. 1-6, Conodonts and graptolites from the Los Guanacos quarry, Salagasta. 1, Lenodus variabilis (Sergeeva). Pb element, anterior view, INGEO-MP-5001 (1). 2-4, Paroistudus horridus (Barnes and Poplawski). 2, P element, lateral view, INGEO-MP-5002 (1). 3, $P$ element, lateral view, INGEO-MP-5002 (2). 4, Sb element, lateral view, INGEO-MP-5002 (3). Scale bar=100 $\mu \mathrm{m}$. 5. Levisograptus $s p$. cf. Levisograptus austrodentatus (Harris and Keble); IANIGLA-PI 2979, mature and incomplete specimen, showing slightly undulating medium septum and sigmoidal thecae with double curvature. Another associated proximal end, poorly preserved, is also present in the same sample. 6. Levisograptus sp.; IANIGLA-PI 2980, incomplete juvenile tubarium. Scale bar $=1 \mathrm{~mm}$. 
Beresi, M.S., et al., 2017. El Darriwiliense inferior (Ordovícico Medio) en el extremo... Boletín Geológico y Minero, 128 (1): $93-110$

las secciones del sur de Salagasta, lo que representa un importante salto bioestratigráfico con el sector norte aquí estudiado. Este fenómeno se verifica también en el norte de la Precordillera en el área de Guandacol-Gualcamayo.

\section{Graptolitos}

Para la clasificación de los graptolitos se tuvieron en cuenta los trabajos de Maletz $(2011,2014)$. A pesar del escaso material recuperado han podido distinguirse dos especies diferentes, correspondientes al género Levisograptus (Maletz, 2011), mientras que para su inclusión en los taxones superiores se ha seguido la reciente propuesta de Maletz (2014).

Phyllum HEMICHORDATA Bateson, 1885

Clase PTEROBRANCHIA Lankester, 1877

Subclase GRAPTOLITHINA Bronn, 1849

Orden GRAPTOLOIDEA Lapworth, 1875 in

Hopkinson and Lapworth, 1875

Familia DIPLOGRAPTIDAE Lapworth, 1873

Género Levisograptus Maletz, 2011

Especie tipo: Fucoides dentatus Brongniart, 1828

Levisograptus sp. cf. Levisograptus austrodentatus (Harris y Keble, 1932)

Figura 4.5

Material.- Dos ejemplares incompletos y regularmente preservados en caliza y numerosos fragmentos aplastados y deformados. Uno de los tubarios ilustrados (Fig. 4.5) corresponde a un ejemplar adulto que conserva parcialmente su relieve se identifica como IANIGLA-PI 2979, y se encuentra asociado con un extremo proximal regularmente preservado.

Descripción.- Este material presenta un extremo proximal achatado y suavemente redondeado. Las primeras tecas se encuentran dispuestas en forma simétrica y puede inferirse en ellas la posición de las espinas aperturales, a pesar de que no se encuentran completas. En el ejemplar adulto de la figura 4 se observa el tubario incompleto, que alcanza $11 \mathrm{~mm}$ de longitud y presenta un septo medio suavemente ondulado. Posee escaso ensanchamiento, alcanzando $1,8 \mathrm{~mm}$ de ancho máximo. Las tecas son delgadas, se encuentran marcadamente traslapadas y poseen una doble curvatura que les confiere un aspecto levemente sigmoidal.

Discusión.- Maletz (2011) incluyó en el género Levisograptus las especies del grupo de Undulograptus austrodentatus (Harris and Keble), diferenciándolas de otros taxones más jóvenes, como Eoglyptograptus gherardi Maletz, con los que fueran previamente confundidas. Nuestro material resulta similar al ilustrado por Toro and Brussa (2003, pl. 13 , figs. $11-12$ y 15), proveniente de la sierra de la Invernada, provincia de San Juan. Recuerda también a los ejemplares localizados por Toro and Brussa (1997) en la sección estratigráfica expuesta entre las localidades de Toquero y Tafna, en el extremo norte de la Puna oriental argentina, que fueron asignados a Undulograptus sp. y mas recientemente a Undulograptus cf. austrodentatus por Toro y Lovalvo (2016). Posee además similar ensanchamiento y espaciamiento tecal que los ejemplares ilustrados por Mitchell (1994) como Undulograptus austrodentatus americanus (Bulman), en su revisión de las especies pertenecientes al grupo de Undulograptus austrodentatus, provenientes de la Formación Fort Peña del oeste de Texas, EEUU. Sin embargo, debido a la escasez del material estudiado y a la imposibilidad de la observación directa de su estructura proximal se prefiere compararlo con la especie mencionada.

\section{Levisograptus sp.}

Figura 4.6

Material.- Un ejemplar juvenil, numerado como IANIGLA-PI 2980.

Observaciones.- El ejemplar presenta similitudes notables con las especies del grupo de Levisograptus austrodentatus, como su extremo proximal con indicios de espinas aperturales en las dos primeras tecas y la presencia de un parche de pirita en la posición en que Maletz (2011, Text-Fig. 2. H, F) ilustra el remanente del manubrio, (Fig. 4.6). Sin embargo, se diferencia de esos taxones por el menor tamaño del tubario, que alcanza solamente $6 \mathrm{~mm}$ de longitud y no supera los $1.1 \mathrm{~mm}$ de ancho. Asimismo, posee menor ancho que el resto de nuestro material asignado a Levisograptus $\mathrm{sp}$. cf. Levisograptus austrodentatus y recuerda a los ejemplares descriptos por Williams (1992) como Undulograptus sp. b, provenientes de Long Island, parte central de Newfoundland. Sin embargo, la escasez y pobre preservación de nuestro material no permiten asegurar una asignación específica.

\section{Trilobites}

Los trilobites hallados se componen de una amplia diversidad de caparazones desarticulados y muy fragmentados que impiden, en la mayoría de los casos, establecer una segura asignación sistemática.

Entre ellos, el taxón más abundante y mejor preservado es el rafiofórido Mendolaspis salagastensis 
Rusconi, 1951. Además, hay escasos restos indeterminados de metagnostidos, illaenidos, harpidos, trinucleidos, encrinuridos, telefinidos y leiostegidos.

\section{Orden AGNOSTIDA Salter, 1864}

Suborden AGNOSTINA Salter, 1864

Superfamilia AGNOSTOIDEA M'Coy, 1849

Familia METAGNOSTIDAE Jaekel, 1909

Género Geragnostus Howell, 1935

Especie tipo: Agnostus sidenbladhi Linnarsson, 1869

\section{Geragnostus? sp.}

Figura 5.8

Discusión.- El único pigidio hallado (IANIGLA-PI: 2968) se asigna con dudas al género Geragnostus? porque no se dispone de céfalos. Por las características del pigidio de forma bien convexa y de contorno circular, con axis de lados paralelos con un nodo pigaxial bien prominente e indicios de un nodo terminal en el pigaxis, campos pleurales con mucha pendiente, con un profundo y ancho surco marginal y borde marginal angosto y con una pequeña espina posterolateral. Se parece al ejemplar ilustrado por Benedetto et al. (1986) de la transición entre las formaciones San Juan y Gualcamayo del área de Guandacol, La Rioja. También tiene semejanza con la forma descripta por Tortello and Peralta (2004) de la Formación Gualcamayo en el cerro La Chilca, San Juan.

\section{Orden CORYNEXOCHIDA Kobayashi, 1935}

Suborden ILLAENINA Hawle and Corda, 1847 Familia ILLAENIDAE Hawle and Corda, 1847

\section{Illaenidae gen. et sp. indet.} Figuras 5.6 y 7

Discusión.- El material, compuesto por varios cranidios y pigidios (IANIGLA-PI: 2970, 2971, 2972, 2973), se ha clasificado en nomenclatura abierta porque no se pueden reconocer los caracteres diagnósticos genéricos ni específicos. Se reconoce la familia Illaenidae según los criterios de Whittington (1997) por la región axial del céfalo liso y que grada hacia delante en forma transicional en un área frontal, sin surcos glabelares ni occipital. El pigidio muy liso con forma semicircular, más ancho que largo de poca convexidad y sin facetas articulares. Pigaxis corto y apenas elevado con surcos axiales someros convergentes hacia atrás. Campos pleurales muy amplios con sus extremos posteriores suavemente inclinados hacia abajo. Se percibe un difuso surco medio en la superficie exfoliada, con un incipiente borde pigidial. Se parece a los pigidios de Platillaenus sp. de la Formación San Juan (Vaccari, 2001) y también posee cierta semejanza a los ejemplares de illaenidae gen. et sp. indet. (Tortello and Peralta, 2004) del cerro La Chilca, San Juan.

\section{Familia LEIOSTEGIIDAE Bradley, 1925 Leiostegiidae gen. et sp. indet. \\ Figura 5.3}

Discusión.- Se halló una mejilla libre (IANIGLA-PI: 2964) que puede ser asignada a esta familia por la ornamentación anastomosada y una bien desarrollada espina genal. Se podría comparar tentativamente con algunas especies del género Annamitella descriptas por Vaccari (1993), que son comunes en la Formación San Juan de Mendoza y San Juan.

Orden PHACOPIDA Salter, 1864

Familia ENCRINURIDAE Angelin, 1854

Encrinuridae? gen. et sp. indet.

Figura 5.1

Discusión.- Se halló un pequeño fragmento de glabela (IANIGLA-PI: 2963) que podría asignarse a esta familia por la forma general y la presencia de un par de surcos glabelares cortos a modo de incisiones longitudinales subparalelas al borde glabelar lateral y por la ornamentación superficial con tubérculos. Representantes de esta familia se reconocen en la Formación Las Aguaditas (Edgecombe et al., 1998).

\section{Orden PROETIDA Fortey y Owens, 1975 \\ Familia TELEPHINIDAE Marek, 1952 Telephinidae gen. et $\mathrm{sp}$. indet.}

Figura 5.2

Discusión.- Se halló un sólo fragmento del aparato ocular (IANIGLA-PI: 2969). Por las características de la superficie ocular convexa integrada por numerosas y pequeñas facetas oculares que poseen contorno hexagonal y agrupadas según un patrón creciente lineal y radial se podrían asignar a esta familia. Representantes de esta familia son comunes en la parte superior de la Formación San Juan y la parte inferior de la Formación Las Aguaditas de la Precordillera sanjuanina (Baldis y Blasco, 1974; Chatterton et al., 1999). 

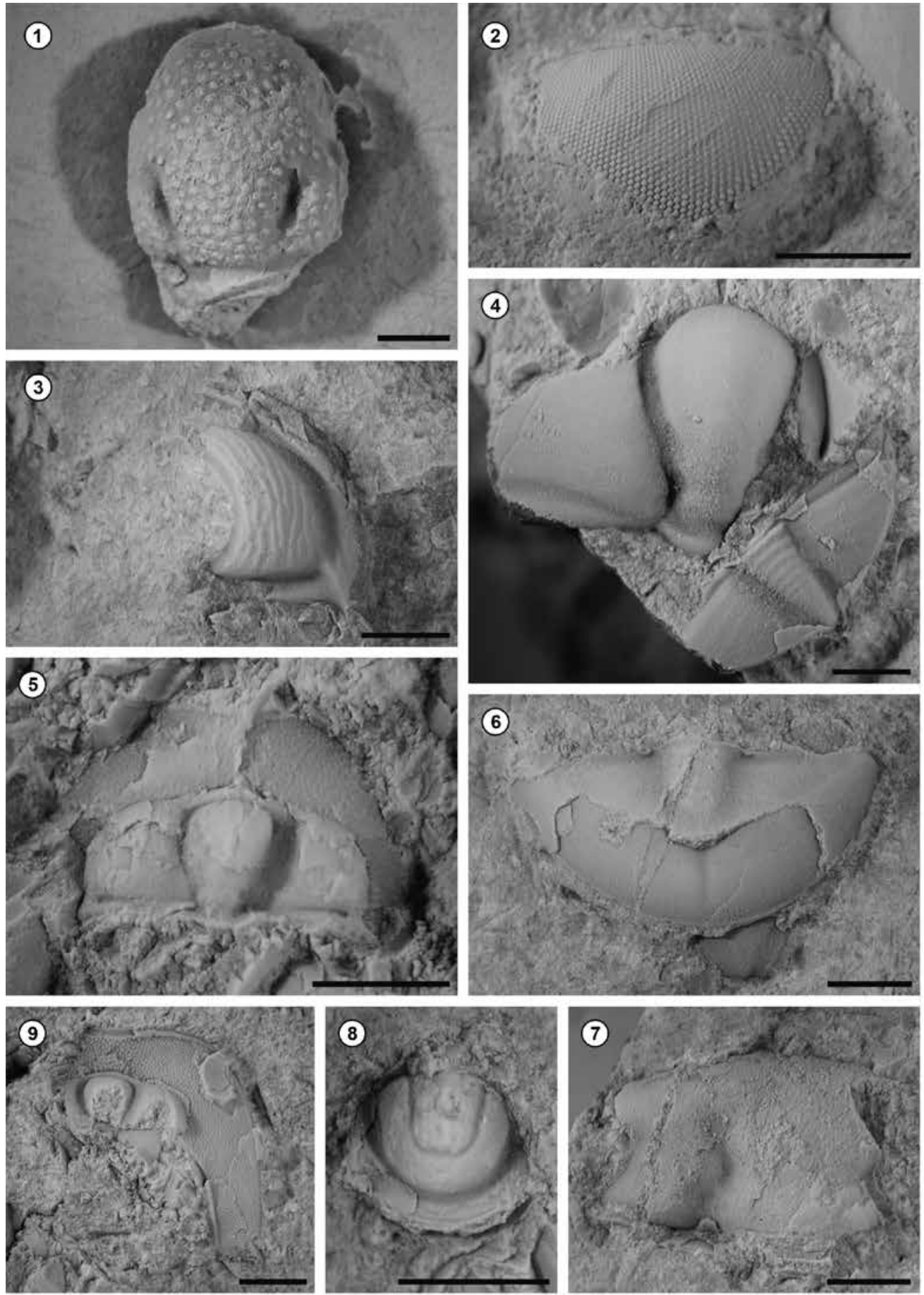

Figura 5. 1-9, Trilobites de la cantera Los Guanacos, Salagasta.1, glabella gen. et sp. indet. de la Familia Encrinuridae (IANIGLA-PI: 2963). 2, fragmento ocular gen. et sp. indet. de la FamiliaTelephinidae (IANIGLA-PI: 2969). 3, Mejilla libre gen. et sp. indet. de la Familia Leiostegiidae (IANIGLA-PI: 2964). 4, cranidio y pigidio de Mendolaspis salagastensis Rusconi (IANIGLA-PI: 2958). 5, Céfalo gen. et sp. indet. de la Familia Trinucleidae (IANIGLA-PI: 2961). 6, Pigidio gen. et sp. indet. de la Familia Illaenidae (IANIGLA-PI: 2970). 7, Céfalo gen. et sp. indet. de la Familia Illaenidae (IANIGLA-PI: 2972). 8, Pigidio de Geragnostus? sp., IANIGLA-PI: 2968. 9, Céfalo gen. et sp. indet. de la FamiliaTrinucleidae, IANIGLA-PI: 2965. Escala gráfica $=0,25 \mathrm{~cm}$.

Figure 5. 1-9, Trilobites from the Los Guanacos quarry, Salagasta. 1, glabella of Encrinuridae gen. et sp. indet. (IANIGLA-PI: 2963). 2, Ocular fragment ofTelephinidae gen. et sp. indet. (IANIGLA-PI: 2969). 3, Free cheek of Leiostegiidae gen. et sp. indet. (IANIGLA-PI: 2964). 4, Cranidium and pygidium of Mendolaspis salagastensis Rusconi (IANIGLA-PI: 2958). 5, Cephalon ofTrinucleidae gen. et sp. indet. (IANIGLAPI: 2961). 6, Pygidium of IIlaenidae gen. et sp. indet. (IANIGLA-PI: 2970). 7, Cephalon of Illaenidae gen. et sp. indet. (IANIGLA-PI: 2972). 8, Pygidium of Geragnostus? sp., (IANIGLA-PI: 2968). 9, Cephalon ofTrinucleidae gen. et sp. indet. (IANIGLA-PI: 2965). Scale bar = $0.25 \mathrm{~cm}$. 
Beresi, M.S., et al., 2017. El Darriwiliense inferior (Ordovícico Medio) en el extremo... Boletín Geológico y Minero, 128 (1): $93-110$

\author{
Orden ASAPHIDA Salter, 1864 emend. Fortey and \\ Chatterton, 1988 \\ Superfamilia TRINUCLEOIDEA Hawle and Corda, \\ 1847 \\ Familia RAPHIOPHORIDAE Angelin, 1854 \\ Género Mendolaspis Rusconi, 1951 \\ Especie tipo: Mendolaspis salagastensis Rusconi, \\ 1951
}

Mendolaspis salagastensis Rusconi

Figuras $6.1,8$ y 5.4

1995 Mendolaspis sanjuaninus Baldis en Baldis et al., 1995

2003 Mendolaspis sanjuaninus Baldis, Waisfeld y Vaccari

2004 Mendolaspis salagastensis Rusconi, Tortello y Peralta (véase sinonimia)

Material.- Se hallaron varios pigidios y cranidios desarticulados y en regular estado de preservación. (IANIGLA-PI: 2957-2960, 2966, 2974-2978)

Discusión.- Harrington and Leanza (1957) hicieron una descripción actualizada de $M$. salagastensis, aunque el deficitario estado de preservación del material usado, proveniente de Guandacol y Rinconada, no les permitió precisar detalles tanto del céfalo como del pigidio. Tortello and Peralta (2004) ilustraron ejemplares procedentes del cerro La Chilca de San Juan con mejor estado de conservación que les permitió detectar cierta variabilidad en el cranidio. Tal como el grado de expresión de los surcos glabelares, un par de fósulas sobre los ángulos anterolaterales de la glabela y un delicado nodo axial por delante del punto medio glabelar. La mayor anchura del cranidio y la del trazo sigmoidal de la sutura facial posterior y un orificio en los ángulos posterolaterales del cranidio. También en el pigidio los últimos autores mostraron un patrón de segmentación variable entre 16 y 19 pares de impresiones musculares dobles dependiendo del grado de conservación. Sobre esta variabilidad detectada no vieron mayores diferencias con $M$. sanjuaninus Baldis en Baldis et al., 1995, excepto en la ausencia de segmentación sobre los campos pleurales, que lo interpretaron de índole preservacional. Waisfeld and Vaccari (2003) consideran que el menor largo sagital en el cranidio y pigidio de $M$. sanjuaninus puede ser debido al crecimiento alométrico, ya que los ejemplares ilustrados por Baldis et al. (1995) son holaspidos juveniles.

El material aquí ilustrado corrobora las apreciaciones de Tortello and Peralta (2004) en lo referido a cranidios (Figs. 6.2 y 7) con un par de fósulas anterolaterales en la glabela y un suave nodo axial por delante del punto medio glabelar. Hay pigidios (Figs. 6.1, 3, 4, 5, 7 y 5.8) en los que se reconoce un patrón de segmentación en el axis del orden de 19 anillos axiales y en ejemplares despojados de su molde externo (Fig. 6.3) se aprecian leves impresiones musculares sigmoidales que están más marcadas en la unión con los surcos axiales. También es posible apreciar en los campos pleurales de algunos moldes internos pigidiales (Figs. 6.3 y 5.8) notables marcas de segmentación que llegan hasta 9 segmentos pleurales levemente sigmoidales que acompañan al diseño sigmoidal del surco anterior. Los pigidios de tamaños pequeños (Figs. 6.4, 6.6) muestran menor largo sagital en relación al ancho transversal. Estas proporciones responden a ejemplares juveniles que comprueban la idea de Waisfeld y Vaccari (2003). Además, donde es posible apreciar el borde pigidial se ve claramente que tiene una pronunciada pendiente adventral (Figs. 6.1, 6.6).

Por los detalles observados en este nuevo material y teniendo en cuenta las apreciaciones de Tortello and Peralta (2004) y de Waisfeld and Vaccari (2003), se considera que las variaciones enunciadas en $M$. sanjuaninus para diferenciarlo de $M$. salagastensis no son diferencias morfológicas válidas, sino que se deben tanto a factores preservacionales como al crecimiento alométrico de ejemplares juveniles comparados con ejemplares adultos. Por lo tanto, $M$. sanjuaninus se considera un sinónimo de $M$. salagastensis.

Horizonte y edad.- En la Provincia de Mendoza $M$. salagastensis fue registrado en el extremo sur de la sierra de Las Higueras (Salagasta), dentro del tramo superior de la Formación San Juan (cantera $n^{\circ} 4$, cantera $n^{\circ} 3$ y $500 \mathrm{~m}$ al sur de cantera $\mathrm{n}^{\circ} 3$ (Rusconi, 1951, 1952; ver figura 1) y su edad se ajusta al Ordovícico Inferior, Floiense, según los conodontes estudiados por Heredia et al. (2009). El presente hallazgo procede del extremo norte de la sierra de Las Higueras (Salagasta), ubicado a unos $5 \mathrm{~km}$ al norte de las localidades referidas originalmente por Rusconi (op.cit.) y se ubica en un nivel calcáreo de la transición a las lutitas negras sobrepuestas a la Formación San Juan, unidad que fue denominada como Formación Gualcamayo (Heredia et al., 2009), o transfacies (Beresi et al., 1998). La edad asignada en esta oportunidad es Ordovícico Medio, Darriwiliense inferior alto, en base a la asociación con conodontos y graptolitos analizados en este trabajo. En cambio, en la Provincia de San Juan, donde tiene una dispersión geográfica y estratigráfica muy amplia, se halla presente en la Formación San Juan de Guandacol y Rinconada (Harrington and Leanza, 1957), en los $4 \mathrm{~m}$ basales de la Formación 

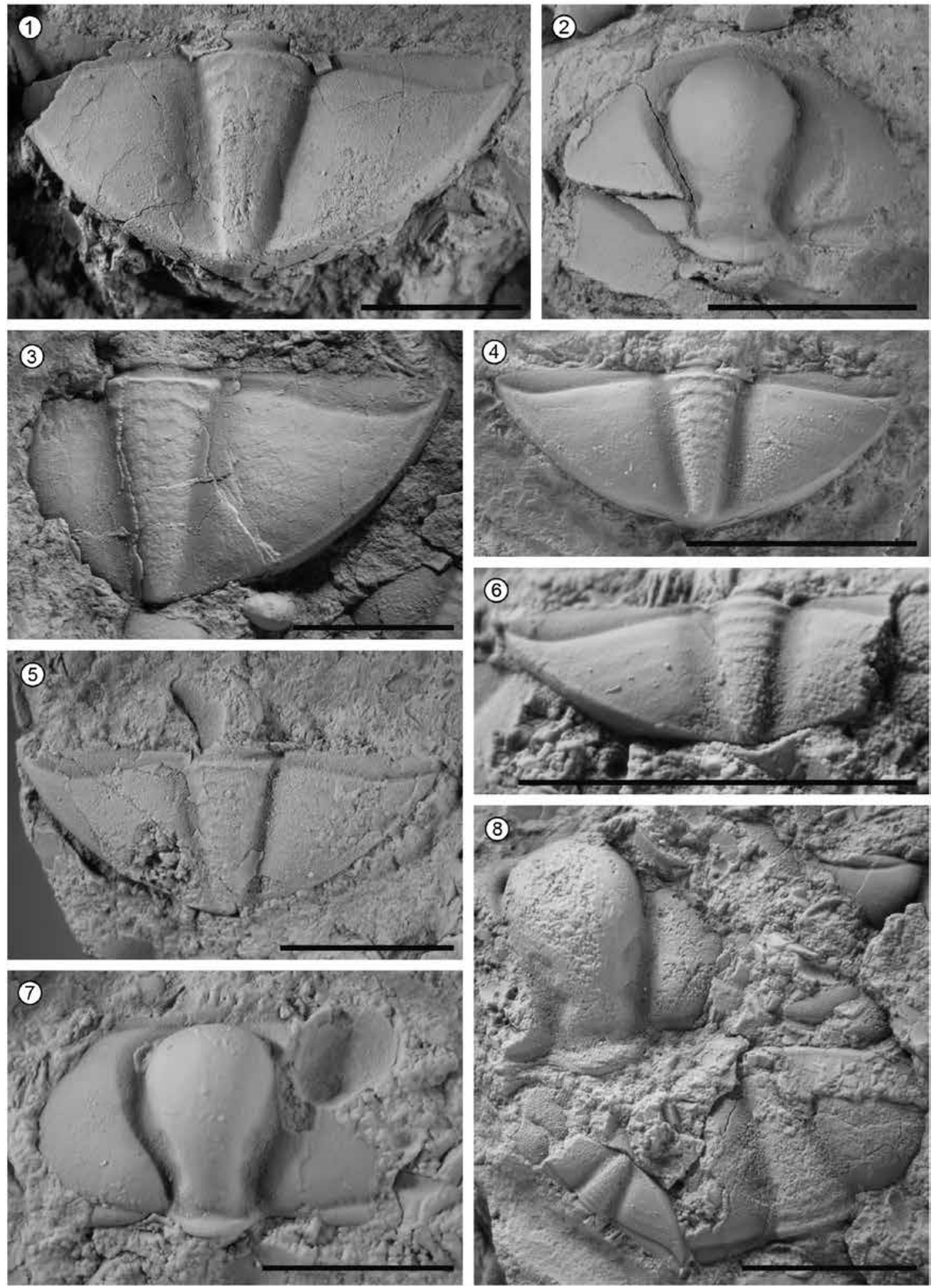

Figura 6. 1-8, Mendolaspis salagastensis Rusconi, de la cantera Los Guanacos, Salagasta. 1, Pigidio IANIGLA-PI: 2966. 2, Cranidio IANIGLAPI: 2962. 3, Pigidio IANIGLA-PI: 2967. 4, Pigidio IANIGLA-PI: 2977. 5, Pigidio IANIGLA-PI: 2957. 6, Pigidio IANIGLA-PI: 2975. 7, Cranidio IANIGLA-PI: 2959. 8, Pigidio IANIGLA-PI: 2975 y cranidio IANIGLA-PI: 2976. Escala gráfica $=0,5 \mathrm{~cm}$.

Figure 6. 1-8, Mendolaspis salagastensis Rusconi, from the Los Guanacos quarry, Salagasta. 1, Pygidium IANIGLA-PI: 2966. 2, Cranidium IANIGLA-PI: 2962. 3, Pygidium IANIGLA-PI: 2967. 4, Pygidium IANIGLA-PI: 2977. 5, Pygidium IANIGLA-PI: 2957. 6, Pygidium IANIGLA-PI: 2975. 7, Cranidium IANIGLA-PI: 2959. 8, Pygidium IANIGLA-PI: 2975 and cranidium IANIGLA-PI: 2976. Scale bar $=0.5 \mathrm{~cm}$. 
Gualcamayo del cerro La Chilca, de Los Azules y de Huaco con una edad Darriwiliense inferior (Tortello and Peralta, 2004; Benedetto et al., 1986), en los 10 $\mathrm{m}$ basales de la Formación Las Aguaditas (Baldis and Pothe de Baldis, 1995; Baldis et al., 1995) en la localidad de Jáchal del Darriwiliense inferior y también en la transición entre la Formación San Juan y en la Formación Los Azules de la sierra de Villicúm [en las Ilamadas "transfacies" por Baldis and Beresi (1981)] con una edad Darriwilliense inferior.

Familia TRINUCLEIDAE Hawle and Corda, 1847 Trinucleidae gen. et sp. indet.

\section{Figuras 5.5 y 5.9}

Discusión.- Se encontraron dos fragmentos de céfalos (IANIGLA-PI; 2965 y 2961) en los que destaca la glabela piriforme expandida hacia delante, que casi toca un angosto borde marginal anterior. Con genas subtriangulares globosas y sin ojos visibles. Acompaña al céfalo una orla bilaminar, amplia y compuesta por numerosos hoyuelos, que se extiende hasta más atrás del borde posterior cefálico y se proyecta en un una gruesa y larga espina cefálica. Varios representantes de esta familia se hallan presentes en la parte inferior de la Formación Las Aguaditas (Baldis and Pothe de Baldis, 1995).

\section{Braquiópodos}

Los fósiles dominantes de la asociación son braquiópodos lingúlidos de diversos tamaños y morfologías que exhiben sus valvas externas desarticuladas y algunas bastante completas, aunque en su mayoría están fragmentadas y no presentan selección por tamaño. Se han observado también, escasos moldes de valvas externas de braquiópodos articulados tipo órthidos de $3 \mathrm{~mm}$ de ancho con una fina estriación radial (Figs.7.4-7.5).

La totalidad de los ejemplares muestra una acusada deformación tectónica y en general presentan un mal estado de preservación de su concha original quitino-fosfática, razón por la cual los fósiles destacan con una coloración azulada a negruzca en la matriz grisácea donde se hallan dispuestos.

Las valvas presentan superficies lisas o estriadas y en algunas se observa la fábrica geopetal con reIleno esparítico y de micrítica pelletoidal. El grano grueso de la roca y la disposición caótica ha dificultado la preservación de detalles morfológicos internos diagnósticos (Figs.7.1-7.2). Sin embargo, una somera descripción acorde a las características externas de las conchas de los braquiópodos linguliformes, permite distinguir claramente dos morfotipos: a) formas grandes, de concha gruesa y exfoliable de contorno subtriangular a subpentagonal con tamaños que varían entre 11 y $12 \mathrm{~mm}$ de máxima longitud y ancho de valvas entre 9 y $12 \mathrm{~mm}$, algunas de las cuales exhiben costulación concéntrica fina; b) valvas de contorno oval a sub-rectangular alargado, con una región anterior bien redondeada, con tamaños que varían entre 2-3 hasta 5-7 mm de largo, algunas con costulación concéntrica fina. Los restos determinables de valvas pertenecen a la Familia Obolidae (Fig. 7, 1-6).

A nivel general, las acumulaciones caóticas de valvas desarticuladas de braquiópodos han sido interpretadas como el resultado del influjo de sedimentos de granos grueso generado por fuertes tormentas estacionales y lluvias que causó la exposición, luego la muerte catastrófica y un rápido enterramiento de los animales (Emig, 1986). Además, se ha argumentado que las valvas de linguliformes, especialmente si están expuestas, tienen un bajo potencial de fosilización ya que se degradan rápidamente al ser desgastados mecánicamente en las zonas mareales y submareales, siendo la porción central altamente mineralizada la que persiste. Esto explica por qué sólo eventos catastróficos conducen a la fosilización de conchas de linguliformes (Emig, op. cit.).

$\mathrm{Li}$ and Droser (1999) clasifican las capas dominadas por braquiópodos-trilobites del Ordovícico, como tipo politaxón (polytaxic), con predominio de órthidos u otros braquiópodos articulados. De acuerdo a esta clasificación, la capa aquí estudiada sería de tipo politaxón, pero con predominio de braquiópodos lingúlidos y trilobites. Aceñolaza et al. (2003) describen concentraciones de conchas de linguliformes tipo monotaxón (monotaxic) del Ordovícico Medio, registradas en diferentes localidades de la Cordillera Oriental del noroeste argentino similares a las que ocurren en las plataformas del SW de Gondwana. Estas capas, denominadas "Lingulate Shell Beds", se caracterizan por tener contactos abruptos con la roca subyacente y por estar relacionadas con eventos sedimentarios excepcionales como tempestitas.

Teniendo en cuenta lo anterior, consideramos que la concentración fosilífera estudiada es de tipo polytaxic, con predominio de braquiópodos lingúlidos y trilobites y fue el resultado de un corto evento catastrófico tipo tempestítico que afectó las comunidades infaunales de la plataforma carbonática somera, en este sector de la Precordillera de Mendoza. 

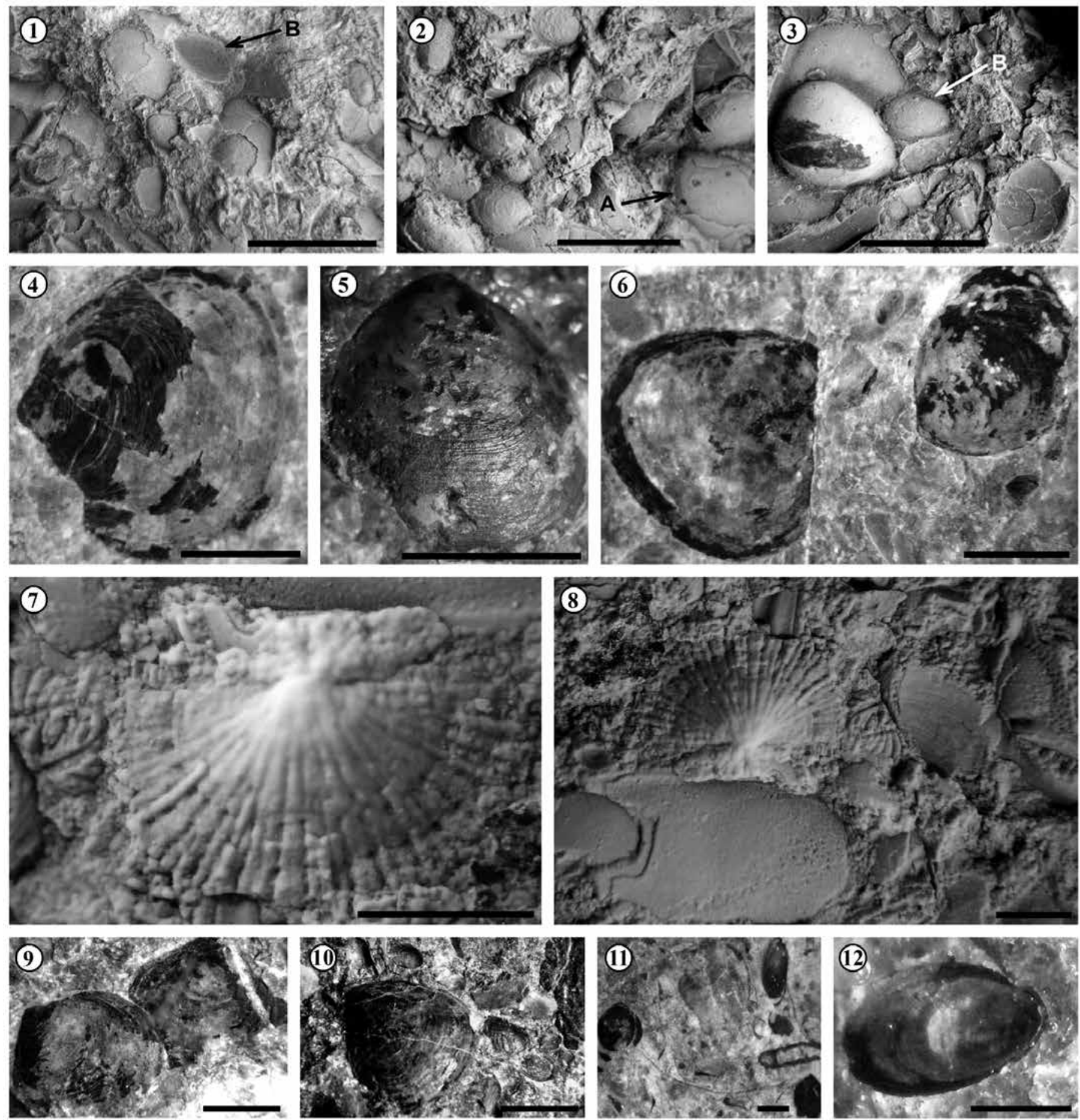

Figura 7. 1-12, Braquiópodos de la cantera Los Guanacos, Salagasta. 1-3, 8, vista del plano superior de la capa bioclástica con braquiópodos linguliformes de diferentes morfotipos (4-6, 9-12); y braquiopodo rhynchonelliforme (7); 3 , note linguliforme morfotipo B superpuesto a cranidio de trilobite; 4-6, 9, 10, valvas externas de linguliformes morfotipo A (Familia Obolidae gen. et sp. indet. A); 7 , vista externa de valva dorsal? de un braquiópodo rhynchoneliforme (¿Orden Orthida?). 8, interior de valva del mismo espécimen; 11-12, braquiópodo linguliforme morfotipo B (Familia Obolidae gen. et sp. indet. B). Escalas gráficas $=5 \mathrm{~mm}(1-6,9,10)$ y $1 \mathrm{~mm}(7,8,11,12)$.

Figure 7. 1-12, Brachiopods from the Los Guanacos quarry, Salagasta. 1-3, 8, overviews of bioclastic bed, showing the different morphotypes of linguliform (4-6, 9-12) and rhynchonelliform brachiopods (7); not: linguliform morphotype B superimposed on trilobite cranidium in 3; 4-6, 9, 10, external views of linguliform morphotype A valves (Family Obolidae gen. et sp. indet. A); 7, external view of dorsal? rhynchonelliform brachiopod valve (Order Orthida?), close-up of 8; 11-12, linguliform morphotype B (Family Obolidae gen. et sp. indet. B). Scale bars $=5 \mathrm{~mm}(1-6,9,10)$ and $1 \mathrm{~mm}(7,8,11,12)$. 


\section{Bioestratigrafía y correlación}

La Zona de Yangtzeplacognathus crassus ha sido identificada en la Formación San Juan en posiciones estratigráficas diferentes (Fig. 8). Se la ha reconocido en la quebrada Del Aluvión (Mestre, 2010; Mestre and Heredia, 2013), en la quebrada de Las Aguaditas (Feltes et al., 2013) y en el borde oriental de la sierra de Villicúm (Mestre, 2013, 2014) en el último metro de la Formación San Juan. En estas secciones el contacto suprayacente con la Formación Los Azules es de carácter transicional y el pase se verifica durante esta biozona, por lo tanto, se podría inferir que la biozona continúa en los primeros metros de la unidad mixta suprayacente. En la quebrada del río de Las Chacritas se registra entre los $4.5 \mathrm{~m}$ y $7 \mathrm{~m}$ aproximadamente desde el tope de esta unidad mixta. En el cerro La Chilca se registran elementos de $Y$. crassus desde $9 \mathrm{~m}$ aproximadamente por debajo del tope, con un espesor estimado de $6.5 \mathrm{~m}$ (Mestre and Heredia, 2013). Por otro lado, en estas dos últimas secciones, la edad del último metro de la Formación San Juan está señalada por el registro de la especie guía Eoplacognathus pseudoplanus (Viira) (Mestre, 2010, 2012; Heredia et al., 2011; Heredia, 2012).
En base a estos datos bioestratigráficos el nivel estudiado en el presente trabajo sería correlacionable con los niveles señalados de la Formación San Juan y los niveles inferiores de la Formación Los Azules (transfacies), y la base de la formación Las Aguaditas, aflorantes en la Precordillera Central y Oriental de la provincia de San Juan (Fig. 8).

Los estudios referidos a los graptolitos de la Precordillera de Mendoza resultan escasos hasta el presente. La presencia de Levisograptus cf. L. austrodentatus y Levisograptus sp. en la localidad de Salagasta confirma por primera vez la existencia de registros del Darriwiliense inferior correspondientes a la Zona de Levisograptus dentatus. La presencia de la especie Levisograptus austrodentatus constituye un importante indicador bioestratigráfico, cuya primera aparición (FAD) fue utilizada para definir la base de la biozona homónima en la sección estratigráfica de Huangnitang, suroeste de China (Mitchell et al., 1995). Sin embargo, resulta muy común también en la suprayacente Zona de Levisograptus dentatus de la Precordillera argentina (Toro and Brussa, 2003; Ortega et al., 2007 y bibliografía allí citada). Por su parte, el ejemplar de Salagasta asignado a Levisograptus sp. se asemeja a Levisograptus sp. b, proveniente de

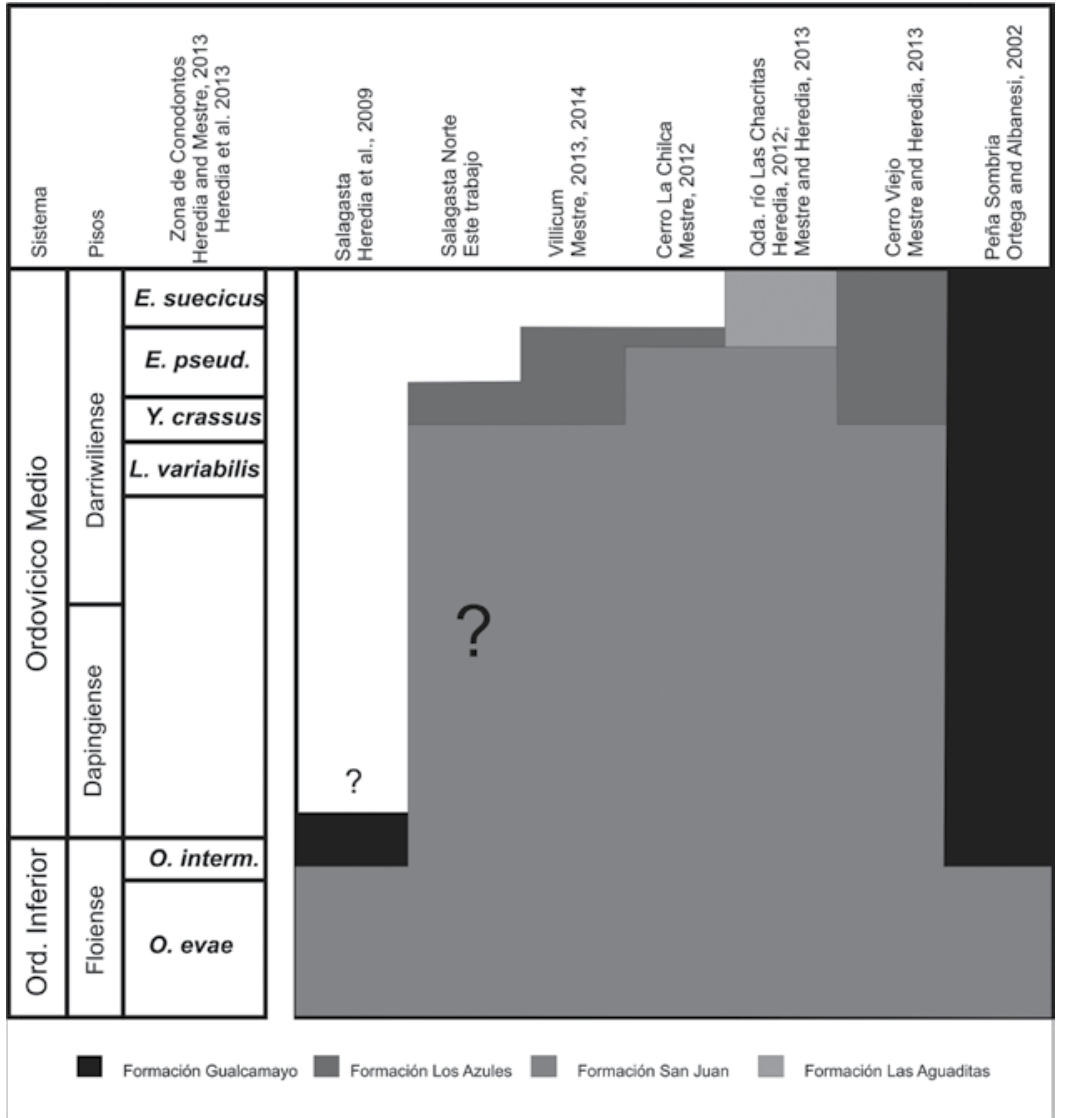

Figura 8. Cuadro de correlación del Ordovícico Inferior y Medio de la Precordillera Argentina.

Figure 8. Correlation chart of the Lower and Middle Ordovician of the Argentine Precordillera. 
niveles equivalentes de Newfoundland (Williams, 1992).

La asociación de la graptofauna estudiada con conodontos de la Zona de Y. crassus permite acotar la edad de los niveles portadores al Darriwiliense inferior alto, correspondiente a la parte superior de la Zona de Levisograptus dentatus e inferior de Yangtzeplacognatus crassus (Voldman et al., 2013: Fig. 3). Asimismo, posibilita la correlación de los estratos portadores con los depósitos inferiores de las formaciones Los Azules y Gualcamayo de la Precordillera de San Juan, en los cuales se desarrolla la Zona de Levisograptus dentatus (Brussa et al., 2003; Ortega et al., 2007), como así también con aquellos de las formaciones Sierra de la Invernada, en la localidad homónima y Las Aguaditas, en el cordón de Los Blanquitos (Brussa et al., 2003).

Una correlación de carácter más regional podría establecerse con los depósitos de la Formación Coquena portadores de Levisograptus cf. L. austrodentatus, en los que Toro y Brussa (1997) encontraron los primeros graptolitos biseriados de la Puna oriental, en el Noroeste argentino.

Los trilobites de la sección estudiada tienen un rango de correlación cronoestratigráfica más amplio que el resto de la fauna, abarcando su edad desde el Floiense superior hasta el Darriwiliense inferior.

\section{Conclusiones}

Se describe en este trabajo, un nivel carbonático de textura packstone/grainstone bioclástico-intraclástico interpretado como el registro parcial de un evento de naturaleza tempestítica, intercalado en la unidad mixta clástica-carbonática que aflora en el extremo norte de la sierra de Las Higueras. Esta unidad mixta (transfacies) que suprayace a las calizas de la Formación San Juan, es correlacionable con la base de la Formación Los Azules en secciones aflorantes en la Precordillera Central y Oriental de la provincia San Juan, oeste de Argentina.

El material fosilífero presenta evidencias de retrabajo y rápida redepositación de los fragmentos y exhiben una importante deformación diagenética, en un ambiente de considerable energía. Se interpreta que el mudstone oscuro basal, corresponde al depósito de post-tormenta, reflejando condiciones de baja energía de la plataforma fangosa. Asimismo, el nivel con estratificación cruzada tipo hummocky podría corresponder al registro de un evento de tormenta anterior al que depositó la capa bioclástica aquí analizada.

Entre los fósiles registrados se han recuperado conodontos y graptolitos asociados a una variada y numerosa fauna de braquiópodos linguliformes de diversos tamaños y morfologías que denotan fragmentación y depositación caótica. Algunos de éstos han sido asignados a la Familia Obolidae.

La presencia de la Zona de Y. crassus permite extender el límite de la cuenca darriwiliense de la Precordillera de San Juan, como también, el notorio cambio sedimentario carbonático a clástico, acontecido durante esa biozona, hasta el sector septentrional de la Precordillera de Mendoza. Este resultado representa, en un contexto regional, un aporte significativo para la reconstrucción de la cuenca de Precordillera durante el Darriwiliense.

Se describen por primera vez los graptolitos localizados en la sierra de Las Higueras, provincia de Mendoza. El material resulta asignable a diferentes especies de Levisograptus, una de las cuales resulta comparable con Levisograptus austrodentatus. Las implicancias bioestratigráficas de estos registros permiten asignarlos a la Zona de Levisograptus dentatus y sugieren una edad darriwiliense temprana alta.

La presencia del trilobites Mendolaspis salagastensis y de restos indeterminados de metagnostidos, illaenidos, harpidos, trinucleidos, encrinuridos, telefinidos y leiostegidos constituyen la primera mención para la localidad de estudio.

\section{Agradecimientos}

Los autores agradecen al Dr. Michael Streng (Universidad de Uppsala, Suecia) por la ayuda brindada en la determinación de los braquiópodos linguliformes. A R. Bottero y R. Marín por la confección de las figuras y a D. Gómez por la preparación de las secciones delgadas y pulidas. A J. Cruz, A. Acosta, y $A$. Menéndez por su colaboración en las tareas de campo. B. Toro agradece el apoyo brindado por el CONICET mediante el subsidio PIP 112-200801-01994. Este trabajo se efectuó en el marco del Proyecto Internacional IGCP 591 y es una contribución al proyecto 653: The onset of the Great Ordovician Biodiversification Event.

\section{Referencias}

Aceñolaza, G.F., Emig, C. and Gutiérrez-Marco, J.C. 2003. Lingulid shell beds from the Ordovician of Argentina, with notes on other peri-Gondwanan occurrences. Serie de Correlación Geológica, 7, 237-244.

Ager, D.V. 1973. Storm deposits in the Jurassic of the Moroccan High Atlas. Palaeogeography, Palaeoclimatology, Palaeoecology, 15, 83-93.

Aigner, T. 1982. Calcareous tempestites: storm-dominated 
Beresi, M.S., et al., 2017. El Darriwiliense inferior (Ordovícico Medio) en el extremo... Boletín Geológico y Minero, 128 (1): $93-110$

stratification in Upper Muschelkalk limenstones (Midle Trias, SW-Germany). In: G. Einsele and A. Seilacher (eds.), Cyclic and Event Stratification. Springer Verlag, Berlin, 180-195.

Baldis, B. y Beresi, M. 1981. Biofacies de culminación del ciclo deposicional calcáreo del Arenigiano en el oeste de Argentina. $2^{\circ}$ Congreso Latinoamericano de Paleontología, Porto Alegre, 2, 11-19.11

Baldis, B. y Blasco, G. 1974. Trilobites ordovícicos de la comarca de Jáchal, Precordillera argentina. 1. Telephinidae. Ameghiniana, 11, 71-87.

Baldis, B. y Pothe de Baldis, D. 1995. Trilobites ordovícicos de la Formación Las Aguaditas (San Juan, Argentina) y consideraciones estratigráficas. Boletín de la Academia Nacional de Ciencias, 60, 409-448.

Baldis, B., Shergold, J. y Peralta, S. 1995. New Llanvirnian trilobites and graptolites from the Las Aguaditas Formation, Argentine Precordillera. $6^{\circ}$ Congreso Argentino de Paleontología y Bioestratigrafía, Actas, Trelew, 31-38.

Benedetto, J.L., Cañas, F.y Astini, R.A. 1986. Braquiópodos y trilobites de la zona de transición entre las Formaciones San Juan y Gualcamayo en el área de Guandacol (La Rioja, Argentina). $4^{\circ}$ Congreso Argentino de Paleontología y Bioestratigrafía, Mendoza, 1, 103-111.

Beresi, M.S. y Rodríguez, M.C. 2011. Microfacies asociadas a las zonas de Oepikodus evae y O. intermedius (conodonta) en el Ordovícico de Salagasta, Mendoza. $10^{\circ}$ Congreso Argentino de Paleontología y Bioestratigrafía y VII Congreso Latinoamericano de Paleontología, La Plata, 43.

Beresi, M.S., Heredia S.E. y Hünicken, M. 1998. El Ordovícico calcáreo de La Sierra de Las Higueras, Salagasta, Precordillera de Mendoza. $7^{\circ}$ Reunión Argentina de Sedimentología, Salta, 15-19.

Beresi, M.S., Bordonaro, O.L., Heredia, S.E. y Mestre, A. 2014. El Darriwiliano inferior (Ordovícico Medio) en el extremo norte de la Sierra de Las Higueras, Salagasta, Precordillera de Mendoza, Argentina. $19^{\circ}$ Congreso Geológico Argentino, Córdoba, S2, 6.

Brussa, E.D., Toro, B.A. y Benedetto, J.L. 2003. Biostratigraphy. In: J.L. Benedetto (ed.), Ordovician Fossils of Argentina. Secretaría de Ciencia y Tecnología, Universidad Nacional de Córdoba, Córdoba, 75-90.

Chatterton, B.D.E., Edgecombe, G.D., Vaccari, N.E. y Waisfeld, B.G. 1999. Ontogenies of some Ordovician Telephinidae from Argentina, and larval pattern in the Proetida. Journal of Paleontology, 73, 219-239.

Dunham, R.J. 1962. Classification of carbonate rocks according to depositional textures. In: Ham, W.P. (ed.), Classification of Carbonate Rocks. American Association of Petroleum Geologists, Memoir 1, Tulsa, 108-121.

Edgecombe, G.D., Chatterton, B.D.E., Waisfeld, B.G. y Vaccari, N.E. 1998. Ordovician (Whiterock) calymenid and encrinurid trilobites from the Precordillera of Argentina. Journal of Paleontology, 72, 678-697.

Emig, C.C. 1986. Conditions de fossilisation du genre Lingula (Brachiopoda) et implications paleoécologiques.
Palaeogeography, Palaeoclimatology, Palaeoecology, $53,245-253$.

Feltes, N.A., Albanesi, G.L. y Bergström, S. 2013. Middle Darriwilian conodont zones in the uppermost San Juan limestone and the Lower Member of the Las Aguaditas Formation, Central Precordillera of San Juan, Argentina. Publicación especial Asociación Paleontológica Argentina, 25-31.

Graves, R.W. y Ellison, S. 1941. Ordovician conodonts of the Marathon Basin, Texas. University of Missouri, School of Mines and Metallurgy, Bulletin 14, 1-26.

Harrington, H.J. y Leanza, A.F. 1957. Ordovician trilobites of Argentina. Department of Geology, University of Kansas Special Publication, 1, Kansas, 276 pp.

Harris, W.J. y Keble, R.A. 1932. Victorian graptolite zones, with correlations and descriptions of species. Proceedings of the Royal Society of Victoria, 44, 25-48.

Heredia, S. 2012. Bioestratigrafía de conodontes del Darriwiliano medio (Ordovícico) de Argentina: la Formación Las Aguaditas, Precordillera Central. Revista Mexicana de Geología, 29, 76-86.

Heredia, S., Beresi, M.S., Mestre, A. y Rodríguez, M.C. 2009. El Ordovícico en la Sierra de La Higuera (Mendoza): conodontes y microfacies. Serie de Correlación Geológica, 25 (1), 7-18.

Heredia, S. y Mestre, A. 2011. Middle Darriwilian Conodont Biostratigraphy in the Argentine Precordillera. In: Gutiérrez-Marco, J.C., Rábano, I. and García Bellido, D. (eds.), Ordovician of the World. Cuadernos del Museo Geominero, 11. Instituto Geológico y Minero de España, Madrid, 229-234.

Heredia, S., Beresi, M.S. y Mestre, A. 2011. La estratigrafía del Ordovícico Medio del río Las Chacritas, Precordillera Central de San Juan. Serie de Correlación Geológica, 27 (1), 18-26.

Heredia, S. y Mestre, A. 2013. Advances in the middle Darriwilian conodont biostratigraphy of the Argentine Precordillera. Publicación Especial de la Asociación Paleontológica Argentina 13, 45 - 48.

Kreisa, R.D. 1981. Storm-generated sedimentary structures in subtidal marine facies with examples from the Middle and Upper Ordovician of Southwestern Virginia. Journal of Sedimentary Petrology, 51, 823-848.

Li, X. y Droser, M.L. 1999. Lower and Middle Ordovician shell beds from the Basin and Range Province of the Western United States (California, Nevada, and Utah). Palaios, 14 (3), 215-233.

Löfgren, A. y Zhang, J. 2003. Element association and morphology in some Middle Ordovician platform equipped conodonts. Journal of Paleontology, 77, 723-39.

Maletz, J. 2011. The identity of the Ordovician (Darriwilian) graptolite Fucoides dentatus Brongniart, 1828. Paleontology, 54 (4), 851-865.

Maletz, J. 2014. The classification of the Pterobranchia (Cephalodiscida and Graptolithina). Bulletin of Geosciences, 89 (3), 477-540.

Mestre, A. 2010. Estratigrafía y bioestratigrafía de conodontes de la "Transición Cuspidal" de la Formación San Juan al sur del paralelo $30^{\circ}$, Precordillera de San Juan. 
Tesis doctoral inédita. Facultad de Ciencias Exactas, Físicas y Naturales de la Universidad Nacional de San Juan, 330 pp.

Mestre, A. 2012. Bioestratigrafía de conodontes del techo de la Formación San Juan y el miembro inferior de la Formación Los Azules, Cerro La Chilca, Precordillera Central. Ameghiniana, 49 (2), 185-197.

Mestre, A. 2013. Middle Darriwilian conodont biostratigraphy of theVillicúm Range, Eastern Precordillera, Argentina. Publicación Especial de la Asociación Paleontológica Argentina 13, 69-72.

Mestre, A. 2014. Bioestratigrafía de conodontos del Darriwiliense medio (Ordovícico) en el borde oriental de la Sierra de Villicúm (Precordillera Oriental, Argentina). Boletín Geológico y Minero, 125 (1), 65-76.

Mestre,A.yHeredia,S.2013.Lazona de Yangtzeplacognathus crassus (conodonta), Darriwiliano de la Precordillera central, San Juan, Argentina. Ameghiniana, 50 (4), 407-417.

Mitchell, C.E. 1994. Astogeny and rhabdosome architecture of graptolites of Undulograptus austrodentatus species Group. In: Chen, X., B., D. Erdtmann and Y. Ni (eds.), Graptolite Research Today. Nanjing University Press, Nanjing, 49-60.

Mitchell, C.E., Maletz, J. y Zhang, Y.D. 1995. Proposal for adoption of the base of the Undulograptus austrodentatus Biozone as a global Ordovician Stage and Series boundary Level. Lethaia, 28, 317-331.

Ortega G., Albanesi, G.L. y Frigerio, S.E. 2007. Graptoliteconodont biostratigraphy and biofacies of the Middle Ordovician Cerro Viejo succession, San Juan Precordillera, Argentina. Palaeogeography, Palaeoclimatology, Palaecology, 245, 245-263.

Rusconi, C. 1951. Fósiles cámbricos de Salagasta. Anales de la Sociedad Científica Argentina, 152, 255-264.

Rusconi, C. 1952. Los fósiles cámbricos de Salagasta. Revista del Museo de Historia Natural de Mendoza, 6, 19-62.

Sergeeva, S.P. 1963. Conodonts from the Lower Ordovician in the Leningrad region [in Russian]. Akademia Nauk SSSR. Paleontologičeskij žurnal, 93-108.

Stouge, S.S. y Nielsen, A.T. 2003. An integrated biostratigraphical analysis of the Volkhov-Kunda (Lower Ordovician) succession at Fågelsång, Scania. Bulletin of the Geological Society of Denmark, 50, 75-94.

Toro, B.A. y Brussa, E.D. 1997. Nuevos hallazgos de graptolitos ordovícicos en la Puna Oriental Argentina. Ameghiniana, 34, 126.

Toro B.A. y Brussa E.D. 2003. Graptolites. En: J. L. Benedetto (ed.), Ordovician fossils of Argentina. Secretaría de Ciencia y Tecnología, Universidad Nacional de Córdoba, Córdoba, 441-505.

Toro, B.A. y Lovalvo, G. 2016. Implicancias bioestratigráficas y correlación de nuevos registros de graptolitos del Ordovícico Inferior y Medio en la transecta ToqueroYavi, provincia de Jujuy, Argentina. $11^{\circ}$ Congreso de la Asociación Paleontológica Argentina. General Roca, Río Negro, Argentina. En prensa.

Tortello, M.F. y Peralta, S.H. 2004. Trilobites del miembro inferior de la Formación Gualcamayo (Llanvirniano temprano) en el cerro La Chilca, Precordillera de San Juan, Argentina. Boletín Geológico y Minero, 115 (4), 665-682.

Vaccari, E.N. 1993. El género Annamitella Mansuy, 1920 (Trilobita, Leiostegiidae) en el Ordovícico de la Precordillera Argentina. Ameghiniana, 30, 395-405.

Vaccari, N.E. 2001. Trilobites de la Formación San Juan (Ordovícico Inferior), Precordillera Argentina. Ameghiniana, 38 (3), 331-347.

Voldman, G.G., Ortega, G. y Albanesi, G.L. 2013. Middle Ordovician conodonts and graptolites at Los Cauquenes range, Central Precordillera of San Juan, Argentina. Publicación Especial Asociación Paleontológica Argentina, 13, 117-121.

Waisfeld, B.G. y Vaccari, N.E. 2003. Trilobites. In: Benedetto, J.L. (ed.), Ordovician fossils of Argentina. Secretaría de Ciencia y Tecnología, Universidad Nacional de Córdoba, Córdoba, 1295-410.

Williams, S.H. 1992. Lower Ordovician (Arenig-Llanvirn) graptolites from the Notre Dame Subzone, central Newfoundland. Canadian Journal of Earth Sciences, 29, 1717-1733.

Recibido: septiembre 2015

Revisado: marzo 2016

Aceptado: abril 2016

Publicado: marzo 2017 